\title{
Inc-PKD2-2-3, identified by long non-coding RNA expression profiling, is associated with pejorative tumor features and poor prognosis, enhances cancer stemness and may serve as cancer stem-cell marker in cholangiocarcinoma
}

\author{
GONGCAI QIU, DONGLAI MA, FUJUN LI, DONGSHENG SUN and ZHAOLIN ZENG \\ Department of Hepatobiliary Surgery, The Second Affiliated Hospital of Harbin Medical University, \\ Harbin, Heilongjiang 150001, P.R. China
}

Received November 7, 2018; Accepted April 22, 2019

DOI: $10.3892 /$ ijo.2019.4798

\begin{abstract}
The present study aimed to explore the long non-coding RNA (IncRNA) expression profiles and correlation of lnc-PKD2-2-3 withtumorfeaturesand prognosis, and toinvestigate its effect on regulating cancer-cell stemness and its potential as a cancer stem cell (CSC) marker in cholangiocarcinoma (CCA). lncRNA expression profiles were determined in 3 pairs of CCA tumors and adjacent tissues by microarray analysis, and lnc-PKD2-2-3 expression was then validated in 60 paired samples by reverse transcription-quantitative polymerase chain reaction (RT-qPCR). Expression of common CSC markers [(CD44, CD133 and octamer-binding transcription factor 4 (OCT4)], $\mathrm{CD} 44^{+} \mathrm{CD} 133^{+}$cell proportions, sphere formation efficiency and drug resistance to 5-fluorouracil (5-FU) were measured following ectopic overexpression of 1nc-PKD2-2-3 or silencing via small hairpin RNA lentivirus transfection into the TFK-1 and Huh-28 CCA cell lines. Finally, lnc-PKD2-2-3 expression was measured in CCA stem-like cells and normal CCA cells. The results from the microarray analysis identified a total of 4,223 upregulated and 4,596 downregulated lncRNAs between CCA tumor tissue and paired adjacent tissue, which were
\end{abstract}

Correspondence to: Dr Zhaolin Zeng, Department of Hepatobiliary Surgery, The Second Affiliated Hospital of Harbin Medical University, 246 Xuefu Road, Harbin, Heilongjiang 150001, P.R. China

E-mail: zzl_zengzhaolin@163.com

Abbreviations: CSCs, cancer stem cells; CCA, cholangiocarcinoma; 5-FU, 5-fluorouracil; HBV, hepatitis B virus; lncRNA, long non-coding RNA; miRNA, microRNA; EMT, epithelialmesenchymal transition; lnc-PKD2-2-3, lncRNA PKD2-2-3; qPCR, quantitative polymerase chain reaction; GO, Gene Ontology; KEGG, Kyoto Encyclopedia of Genes and Genomes; OS, overall survival; FBS, fetal bovine serum; MOI, multiplicity of infection; C-Caspase-3, cleaved Caspase-3; Bcl-2, B-cell lymphoma-2.

Key words: lncRNA expression profile, cholangiocarcinoma, long non-coding RNA PKD2-2-3, stemness, cancer stem cells enriched in regulating cancer-associated pathways. RT-qPCR validation revealed that lnc-PKD2-2-3 was upregulated in CCA and associated with a higher Eastern Cooperative Oncology Group performance score, poor differentiation, advanced TNM stage, increased carcinoembryonic antigen and poor overall survival in CCA patients. In vitro, lnc-PKD2-2-3 increased CD44,CD133 and OCT4 expression as well as the CD44 ${ }^{+} \mathrm{CD} 133^{+}$ cell proportion, raised the sphere formation efficiency and enhanced drug resistance to 5-FU in TFK-1 and Huh-28 cells. In addition, lnc-PKD2-2-3 was positively correlated with CSC markers in CCA tumor tissues and was markedly upregulated in CCA stem-like cells compared with that in normal CCA cells. In conclusion, lnc-PKD2-2-3, selected by lncRNA expression profiling, was associated with pejorative tumor features and poor prognosis, enhanced cancer stemness and may serve as a CSC marker in CCA.

\section{Introduction}

Cholangiocarcinoma (CCA), comprising intrahepatic, perihilar and distal CCA, is an uncommon but lethal cancer type with increasing incidence and mortality worldwide; it is a malignancy that poses a great threat to human health and life $(1,2)$. In Asia, CCA has an even higher incidence and mortality compared with those in other regions due to higher prevalence of hepatitis B virus (HBV) infection and cirrhosis, as well as less advanced medical technology $(3,4)$. Although numerous improvements have been made during recent decades, including novel cancer markers, advanced surgical techniques and skills and novel targeted drugs, the prognosis of patients with CCA remains poor due to late diagnosis, high probability of peripheral vascular, liver and lymph node metastasis, and increased risk of recurrence (5-7). Thus, it is of critical importance to explore the molecular mechanisms associated with the pathogenesis of CCA, and to explore novel treatment targets for CCA to improve the prognosis of affected patients.

Long non-coding RNAs (lncRNAs), a group of endogenous RNAs of $>200$ nucleotides in length with no protein-coding function, are implicated in numerous processes, including epigenetic gene expression regulation, gene imprinting, 
transcriptional activation, mRNA modification, nuclear transportation and protein activation $(8,9)$. Dysregulated IncRNA patterns have been observed to be implicated in the development of various cancers, and certain specific oncogenic or tumor suppressor IncRNAs have been discovered in numerous cancer types, which regulate cancer cell proliferation, apoptosis, migration, invasion and drug resistance via sponging target microRNAs (miRNAs) or binding with cancer-associated mRNAs (10-12). In particular, certain lncRNAs [e.g. lnc-FEZ family zinc finger 1 (FEZF1)-antisense 1 (AS1) and lnc-THOR] have been reported to regulate cancer cell stemness via affecting cancer stem cell (CSC) markers, including sex-determining region $\mathrm{Y}$ box 9 (SOX9), CD44 and Nanog homeobox (NANOG) (13-15). Since CSCs are considered to be the a crucial component of cancer metastasis and recurrence, two processes that are associated with poor prognosis $(16,17)$, these results indicate the potential of specific lncRNAs as treatment targets in cancer. As for CCA, limited data are available regarding the role of lncRNAs and only a small number of IncRNAs have been identified to be implicated in its pathogenesis. For instance, lncRNA EPIC1 was reported to promote CCA cell growth and colony formation while repressing apoptosis via regulating Myc (18). Furthermore, IncRNA ArfGAP with SH3 domain, ankyrin repeat and PH domain 1-intronic transcript 1 was indicated to promote CCA cell proliferation, migration, invasion and epithelial-mesenchymal transition via the hedgehog signaling pathway (19). In addition, IncRNA SOX2 overlapping transcript was reported to stimulate CCA cell proliferation and invasion, and to be associated with poor prognosis in CCA patients (20). However, the functions of most lncRNAs in the pathogenesis of CCA remain largely elusive, and therefore further investigation is required.

The present study aimed to explore the lncRNA expression profiles in CCA by microarray analysis. The results of the microarray analysis identified lncRNA PKD2-2-3 (lnc-PKD2-2-3) as a key lncRNA in CCA. The aberrant expression of lnc-PKD2-2-3 in CCA compared with normal tissues was then confirmed by reverse transcription-quantitative polymerase chain reaction (RT-qPCR). The association of lnc-PKD2-2-3 with tumor features and patient prognosis was determined. Finally, the potential role of lnc-PKD2-2-3 in regulating stemness of CCA cells and its application as a CSC marker in CCA were assessed in in vitro experiments.

\section{Materials and methods}

Patients and samples. A total of 60 consecutive CCA patients treated at the Second Affiliated Hospital of Harbin Medical University (Harbin, China) between January 2014 and December 2015 were enrolled in the present study. The tumor and paired adjacent tissues were obtained during the surgery and immediately stored in liquid nitrogen. The inclusion criteria were as follows: i) Diagnosis as primary CCA according to clinical and pathological findings; ii) age $>18$ years; iii) the patient was scheduled for resection. Patients with prior neoadjuvant therapies were excluded. The present study was approved by the Ethics Committee of the Second Affiliated Hospital of Harbin Medical University (Harbin, China) and all patients provided written informed consent prior to enrollment.
The patients' characteristics were recorded following enrollment and included the following: Age, sex, smoking status, drinking status, HBV infection status, Eastern Cooperative Oncology Group (ECOG) performance score, tumor site, tumor size, number of tumors, degree of tumor differentiation, tumor-nodes-metastasis (TNM) stage, carcinoembryonic antigen (CEA), carbohydrate antigen 199 (CA199) levels and surgery type. Furthermore, patients were followed up until the end of June 2018 with a median follow-up duration of 27.5 months, and the overall survival (OS) time was determined as the time of resection to the time of death.

Microarray and bioinformatics analyses. A total of 3 pairs of CCA tumor tissue and adjacent tissue were randomly selected from all samples and total RNA was extracted using TRIzol ${ }^{\mathrm{TM}}$ reagent (Invitrogen; Thermo Fisher Scientific, Inc.) followed by quantification using a NanoDrop-2000 (Thermo Fisher Scientific, Inc.) and integrity assessment using an Agilent Bioanalyzer 2100 (Agilent Technologies, Inc.). IncRNA and mRNA profiles were then detected using a IncRNA and mRNA microarray kit (Agilent Human lncRNA 4x180K microarray; Agilent Technologies, Inc.) according to the manufacturer's protocol. lncRNAs present in $>50 \%$ of samples were included in the bioinformatics analysis using R software (version 3.3.3). A volcano plot was drawn by dysregulated lncRNAs using the limma package with statistical significance defined as $\mathrm{P}<0.05$ and a fold change $>2.0$. Heatmap analysis of dysregulated IncRNAs was performed using the Pheatmap package. Gene Ontology (GO) and Kyoto Encyclopedia of Genes and Genomes (KEGG) enrichment analysis of dysregulated lncRNA were performed using the database for annotation, visualization and integrated discovery (DAVID) web server (https://david.ncifcrf.gov) $(21,22)$ based on correlated mRNA expression.

Validation of lnc-PKD2-2-3 expression by RT-qPCR. lnc-PKD2-2-3 was one of the most upregulated lncRNAs according to the microarray detection. Inc-PKD2-2-3 targets were then identified by Pearson correlation coefficient, and enrichment analysis was performed using the target genes with DAVID (https://david.ncifcrf.gov) $(21,22)$. This bioinformatics analysis revealed that lnc-PKD2-2-3 was correlated with several oncogenes and stemness-associated genes. Thus, its expression was further assessed in 60 pairs of CCA tumor tissue and adjacent tissue by using RT-qPCR. Apart from the comparison of its expression between tumor tissue and adjacent tissue, the association of lnc-PKD2-2-3 with clinicopathological features as well as OS was analyzed.

Measurement of the correlation between Inc-PKD2-2-3 and CSC markers in CCA tissues. In order to determine the association of lnc-PKD2-2-3 with tumor stemness, the expression levels of CSC markers CD44, CD133 and octamer-binding transcription factor 4 (OCT4) were detected in 60 CCA tumor tissues by RT-qPCR, and the correlation of lnc-PKD2-2-3 with CD44, CD133 and OCT4 levels was analyzed by Spearman analysis.

Cell sources and culture. The human CCA cell line TFK-1 was purchased from the German Collection of Microorganisms and Cell Cultures GmbH (Braunschweig, Germany), while 
the human CCA cell line Huh-28 was purchased from the Japanese Cancer Research Bioresources Bank Cell Bank (Tokyo, Japan). TFK-1 cells were cultured in 90\% RPMI-1640 medium (Gibco; Thermo Fisher Scientific, Inc.) and 10\% fetal bovine serum (FBS; Gibco; Thermo Fisher Scientific, Inc.), while Huh-28 cells were cultured in 80\% RPMI-1640 medium and $20 \%$ FBS. Cell culture was performed in a humidified atmosphere of $95 \%$ air and $5 \% \mathrm{CO}_{2}$ at $37^{\circ} \mathrm{C}$.

Lentivirus construction. Lentiviruses were constructed by Shanghai Gene Bio-Tech Co. In brief, shuttle plasmids for lnc-PKD2-2-3 overexpression and short hairpin (sh) RNA, as well as nonsense DNA fragment overexpression and nonsense DNA fragment shRNA negative control (NC) plasmids, were constructed using pLV1-CMV and pGLV-U6 plasmids, respectively, and then transferred into $293 \mathrm{~T}$ cells along with envelope plasmids and package plasmids. Subsequently, the resulting lentivirus was obtained by collecting the cell supernatant at 48 and $72 \mathrm{~h}$ following transfection.

Lentivirus transfection. Control overexpression, lnc-PKD2-2-3 overexpression, control shRNA and lnc-PKD2-2-3 shRNA lentiviruses were transfected into TFK-1 and Huh-28 cells at a multiplicity of infection of 20 and cultured for $72 \mathrm{~h}$. The cells in the different transfection groups were respectively named as the LV-NC group, LV-Lnc group, LVU6-NC group and LVU6-Lnc group. 1nc-PKD2-2-3 expression was detected by RT-qPCR to determine transfection efficiency. The cells were then cultured with $8 \mu \mathrm{g} / \mathrm{ml}$ puromycin (Sigma-Aldrich; Merck KGaA) for 9 days to obtain stably transfected TFK-1 and Huh-28 cells.

CSC marker expression and $\mathrm{CD} 44^{+} \mathrm{CD} 133^{+}$cell proportion. In order to explore the effect of lnc-PKD2-2-3 on regulating CCA stemness, expression levels of CSC markers CD44, CD133 and OCT4 were detected by qPCR and western blot analysis in each group at $72 \mathrm{~h}$ post-transduction. In addition, $\mathrm{CD} 44^{+} \mathrm{CD} 133^{+}$ cell proportions at $72 \mathrm{~h}$ were detected by flow cytometry using a FACSCalibur (BD Biosciences) and analyzed using Flowjo Software 7.6 (FlowJo, LLC). The antibodies used in flow cytometry were CD133 mouse monoclonal antibody (cat. no. 38725, flow-specific; Alexa Fluor ${ }^{\circledR}$ 488-conjugated; Cell Signaling Technology, Inc.) and CD44 rat monoclonal antibody (cat. no. 80813; allophycocyanin-conjugated; Cell Signaling Technology, Inc.). In brief, cells were harvested, washed with $\mathrm{PBS}$, resuspended in PBS/ $0.5 \%$ bovine serum albumin, then incubated with CD44 rat monoclonal antibody (dilution 1:160) and CD133 mouse monoclonal antibody (dilution 1:50) at room temperature for $1 \mathrm{~h}$. Then the cells were collected, suspended in PBS and detected by flow cytometry.

Measurement of sphere formation efficiency. In order to validate the effect of lnc-PKD2-2-3 in regulating CCA stemness, sphere formation efficiency was detected in each group of transfected cells using a sphere formation assay. In brief, transfected CCA cells were cultured in Dulbecco's modified Eagle's/F12 medium supplemented with 2\% B27 (both from Gibco; Thermo Fisher Scientific, Inc.), $20 \mathrm{ng} / \mathrm{ml}$ epidermal growth factor (Sigma-Aldrich; Merck KGaA), $20 \mathrm{ng} / \mathrm{ml}$ basic fibroblast growth factor (Gibco; Thermo Fisher Scientific, Inc.) and $4 \mu \mathrm{g} / \mathrm{ml}$ heparin (Sigma-Aldrich; Merck KGaA ) for 10 days, and the spheres with a diameter of $>50 \mu \mathrm{m}$ were counted under an inverted fluorescence microscope (Olympus) at x200 magnification. The sphere formation efficiency was calculated as the number of these spheres divided by the total number of seeded cells.

Measurement of resistance to 5-fluorouracil (5-FU). In order to further examine the effect of lnc-PKD2-2-3 in regulating CCA stemness, drug resistance of transduced CCA cells to 5-FU was investigated by measuring cell viability and apoptosis following 5-FU treatment. In brief, $200 \mathrm{ng} / \mathrm{ml} 5-\mathrm{FU}$ was added in the medium of the transduced CCA cells, resulting into four treatments groups: $\mathrm{LV}-\mathrm{NC}+5-\mathrm{FU}$ group, $\mathrm{LV}-\mathrm{Lnc}+5-\mathrm{FU}$ group, LVU6-NC + 5-FU group and LVU6-Lnc + 5-FU group. After $24 \mathrm{~h}$ of incubation, the cell viability was measured using a Cell Counting Kit-8 (CCK-8; Sangon Biotech Co., Ltd.) according to the manufacturer's protocol with measurement of the absorbance under a microplate reader (BioTek Instruments, Inc.). The cell viability of LV-Lnc $+5-\mathrm{FU}$ group was calculated relative to the $\mathrm{LV}-\mathrm{NC}+5-\mathrm{FU}$ group, while the cell viability of LVU6-Lnc +5 -FU group was calculated relative to the LVU6-NC + 5-FU group. The cell apoptosis rate was measured using a fluorescein isothiocyanate Annexin V apoptosis detection kit II with a flow cytometer (both from BD Biosciences) according to the manufacturer's protocol and analyzed using Flowjo Software 7.6 (FlowJo, LLC). Furthermore, the expression of apoptotic markers [cleaved caspase-3 (c-caspase-3) and B-cell lymphoma-2 (Bcl-2)] were measured by western blot analysis.

Measurement of lnc-PKD2-2-3 expression in CCA stem-like cells. Drug-resistant TFK-1 and Huh-28 cells were generated using 5-FU (Sigma-Aldrich; Merck KGaA) with repeated treatment. In brief, cells were cultured in medium containing $200 \mathrm{ng} / \mathrm{ml} \mathrm{5-FU}$ for $72 \mathrm{~h}$ and in the second step, cultured in 5 -FU-free medium for another $72 \mathrm{~h}$. These processes were repeated until no effect of 5-FU on cell viability was observed by using the CCK-8 assay. The drug-resistant cell lines were referred to as R-TFK-1 and R-Huh-28. The sphere formation assay was then performed as aforementioned. Spheres were isolated by centrifugation to obtain samples referred to as S-TFK-1 and S-Huh-28, which served as CCA stem-like cells that were validated by measurement of CSC marker expression CD44, CD133 and OCT4, using RT-qPCR and western blotting, with parental normal CCA cells (TFK-1 and Huh-28) as a reference. Finally, lnc-PKD2-2-3 expression in CCA stem-like cells (S-TFK-1 and S-Huh-28) and normal CCA cells (TFK-1 and Huh-28) was measured using RT-qPCR.

$R T-q P C R$. After extraction of total RNA by TRIzol ${ }^{\mathrm{TM}}$ reagent (Invitrogen; Thermo Fisher Scientific, Inc.) from tissues or cells, quality control was performed with a NanoDrop-2000 (Thermo Fisher Scientific, Inc.) and Agilent Bioanalyzer 2100 (Agilent Technologies, Inc.). The RNA was reverse transcribed into complementary DNA using a PrimeScript ${ }^{\mathrm{TM}}$ RT reagent kit (Perfect Real Time; Takara Bio Inc.) and qPCR was performed using SYBR ${ }^{\circledR}$ Green Real-time PCR master mix (Toyobo Life Science). The thermocycling conditions were as follows: $95^{\circ} \mathrm{C}$ for $5 \mathrm{~min}$, then 40 cycles of $94^{\circ} \mathrm{C}$ for $30 \mathrm{sec}, 61^{\circ} \mathrm{C}$ 
Table I. Primers used for quantitative PCR.

\begin{tabular}{lll}
\hline Gene & \multicolumn{1}{c}{ Forward Primer (5'-3') } & \multicolumn{1}{c}{ Reverse Primer $\left(5^{\prime}-3^{\prime}\right)$} \\
\hline lnc-PKD2-2-3 & AGGCTGATTCTGGAAGTTCTGAG & AGGAGATTCTGCTTCTGAGATGG \\
CD44 & ACATCCTCACATCCAACACCTC & CCTCCTGAAGTGCTGCTCCT \\
CD133 & GCTGCTTGTGGAATAGACAGAATG & GAAGGACTCGTTGCTGGTGAAT \\
OCT4 & AAGCGATCAAGCAGCGACTA & CAGAGTGGTGACGGAGACAG \\
GAPDH & GAGTCCACTGGCGTCTTCAC & ATCTTGAGGCTGTTGTCATACTTCT
\end{tabular}

lnc-PKD22-2-3, long non-coding RNA PKD2-2-3; OCT4, octamer-binding transcription factor 4.

Table II. Antibodies used for western blotting.

\begin{tabular}{|c|c|c|c|}
\hline Antibody & Company & Catalog no. & Dilution \\
\hline \multicolumn{4}{|l|}{ Primary } \\
\hline Rabbit polyclonal to caspase- 3 & Abcam & ab90437 & $1: 2,000$ \\
\hline Rabbit polyclonal to cleaved caspase- 3 & Abcam & ab49822 & $1: 2,000$ \\
\hline Rabbit polyclonal to $\mathrm{Bcl}-2$ & Abcam & ab194583 & $1: 2,000$ \\
\hline Rabbit polyclonal to CD44 & Abcam & ab157107 & $1: 2,000$ \\
\hline Rabbit polyclonal to CD133 & Abcam & ab19898 & $1: 2,000$ \\
\hline Rabbit polyclonal to OCT4 & Abcam & ab19857 & $1: 2,000$ \\
\hline Rabbit monoclonal to GAPDH & Abcam & ab9485 & $1: 2,000$ \\
\hline \multicolumn{4}{|l|}{ Secondary } \\
\hline Goat anti-rabbit (horseradish peroxidase-conjugated) & Abcam & ab6721 & $1: 4,000$ \\
\hline
\end{tabular}

Bcl-2, B-cell lymphoma-2; OCT4, octamer-binding transcription factor 4.

for $30 \mathrm{sec}$. Relative fold changes in mRNA expression were calculated using the formula $2^{-\Delta \Delta \mathrm{Cq}}(23)$ with GAPDH serving as the internal reference. The sequences of the primers are provided in Table I.

Western blot analysis. Total protein was extracted using radioimmunoprecipitation assay lysis buffer (Thermo Fisher Scientific, Inc.) and the protein concentration was measured and adjusted with a bicinchoninic acid kit for protein determination (Sigma-Aldrich; Merck KGaA). A total of $20 \mu \mathrm{g}$ protein per lane was then separated using $10 \%$ Bis-Tris protein gels (Invitrogen; Thermo Fisher Scientific, Inc.), followed by transfer onto a polyvinylidene fluoride membrane (EMD Millipore). Subsequently, the membrane was blocked in $5 \%$ skimmed milk at $37^{\circ} \mathrm{C}$ for $90 \mathrm{~min}$, and then incubated with primary antibody at $4^{\circ} \mathrm{C}$ overnight, followed by incubation with secondary antibody at room temperature for $1 \mathrm{~h}$. Finally, visualization of blots was performed using highly sensitive enhanced chemiluminescence reagent (Sangon Biotech Co., Ltd.) and exposed to X-ray films. GAPDH was used as an internal reference. The antibodies are listed in Table II.

Statistical analysis. Statistical analysis was performed using SPSS 22.0 Software (IBM Corporation) and GraphPad Prism software 6.01 (GraphPad Inc.). Values are expressed as the mean \pm standard deviation or median (25th-75th value) Comparison of paired data between two groups was performed using a Wilcoxon signed rank-sum test. Comparison of individual data between two groups was performed using a t-test or Wilcoxon rank-sum test. Correlations were determined using Spearman analysis. OS between two groups was compared using Kaplan-Meier curves and the log-rank test. $\mathrm{P}<0.05$ was considered to indicate statistical significance.

\section{Results}

Dysregulated IncRNA profiles in CCA determined by microarray analysis. A total of 46,846 lncRNAs were detected in $>50 \%$ samples and included in the analysis, among which 4,223 were upregulated and 4,596 were downregulated in CCA tumor tissues compared with paired adjacent tissues (Fig. 1A). The top 10 upregulated and top 10 downregulated lncRNAs are listed in Table SI. Heatmap analysis revealed that these up and downregulated lncRNAs were able to distinguish CCA tumor tissue from paired adjacent tissue (Fig. 1B). GO enrichment analysis indicated that dysregulated lncRNAs were enriched in GO terms in the categories molecular mechanism (including calcium-dependent protein binding, cell adhesive protein binding and nitric oxide binding), cellular component [including extracellular exosome, cell surface and extracellular matrix (ECM)] and biological process (including complement activation, lectin pathway, ECM organization and cell adhesion; Fig. 1C). KEGG enrichment analysis revealed that the dysregulated lncRNAs were enriched in pathways involving 

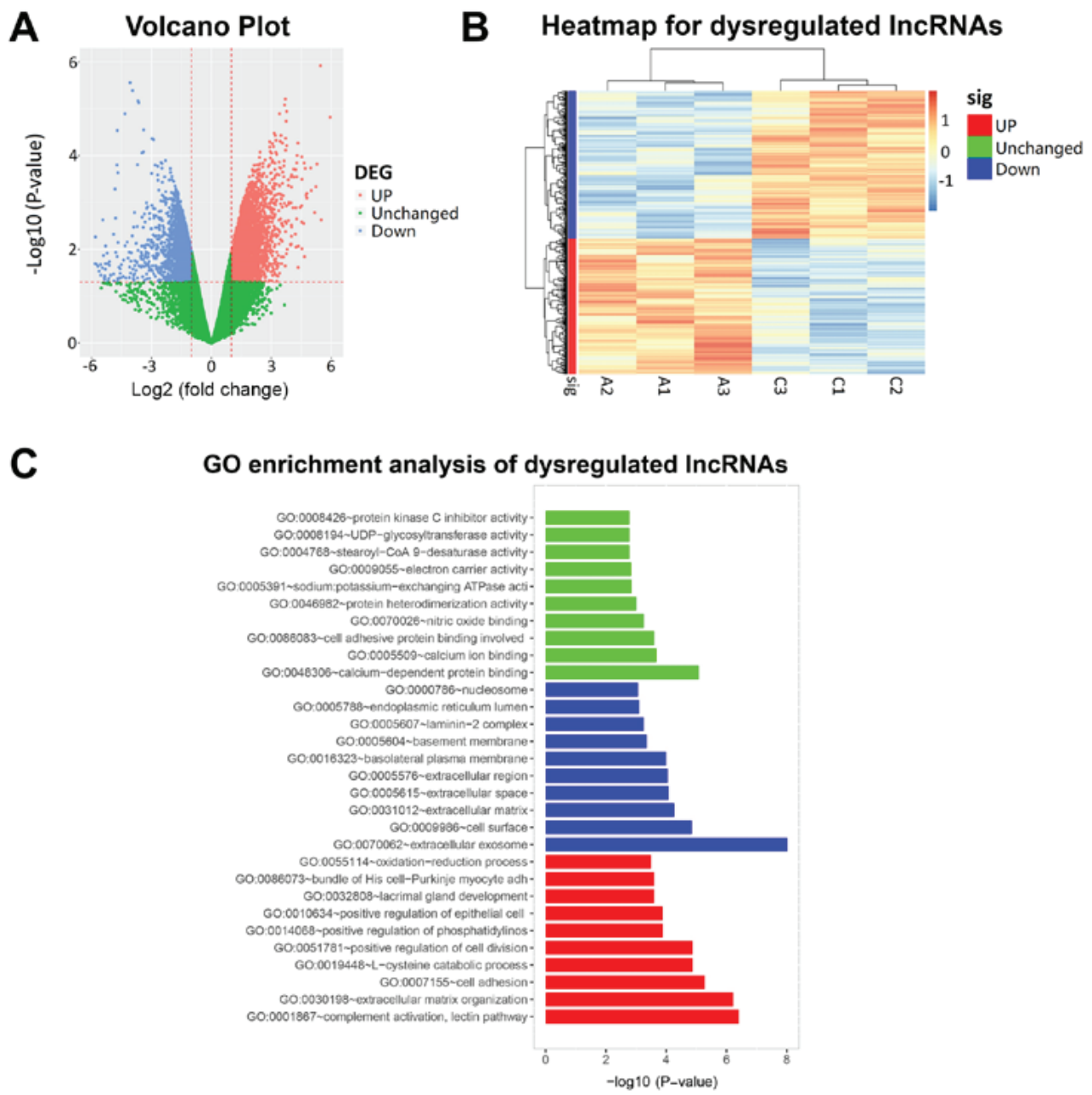

DEGG enrichment analysis of dysregulated IncRNAs

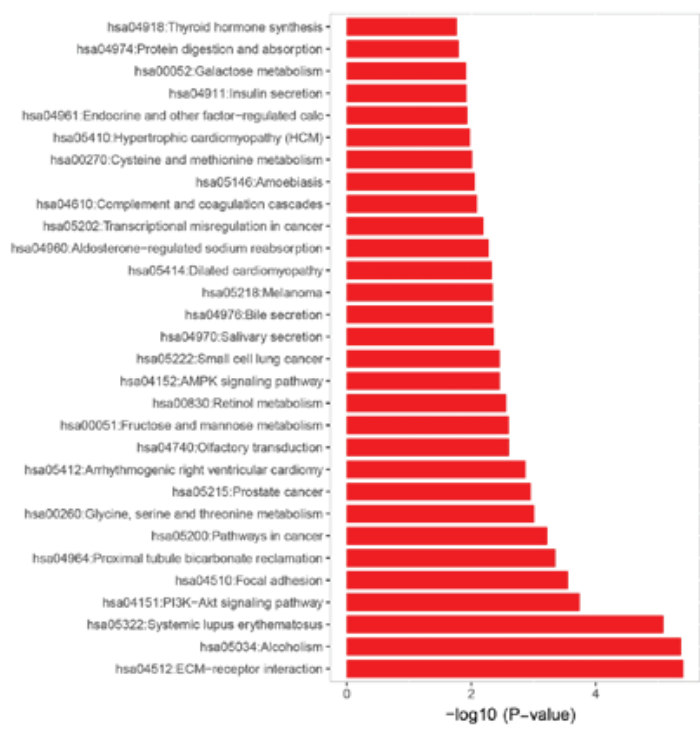

Figure 1. Bioinformatics analysis of microarray. (A) Volcano plot. Red spots indicate upregulated lncRNAs, green spots indicate unchanged lncRNAs, and blue spots indicate downregulated lncRNAs. (B) Heatmap analysis of dysregulated lncRNAs. (C) GO enrichment analysis of dysregulated lncRNAs. (D) KEGG enrichment analysis of dysregulated lncRNAs. IncRNA, long non-coding RNA; GO, Gene Ontology; KEGG, Kyoto Encyclopedia of Genes and Genomes; DEG, differentially expressed gene.

ECM-receptor interaction, alcoholism, PI3K/Akt signaling and pathways in cancer (Fig. 1D). These results indicated that the identified dysregulated lncRNAs may have critical roles in CCA.
Association of lnc-PKD2-2-3 expression with clinicopathological features and prognosis in CCA patients. lnc-PKD2-2-3 was one of the most upregulated lncRNAs in the microarray data (Table SI). In addition, bioinformatics results, 

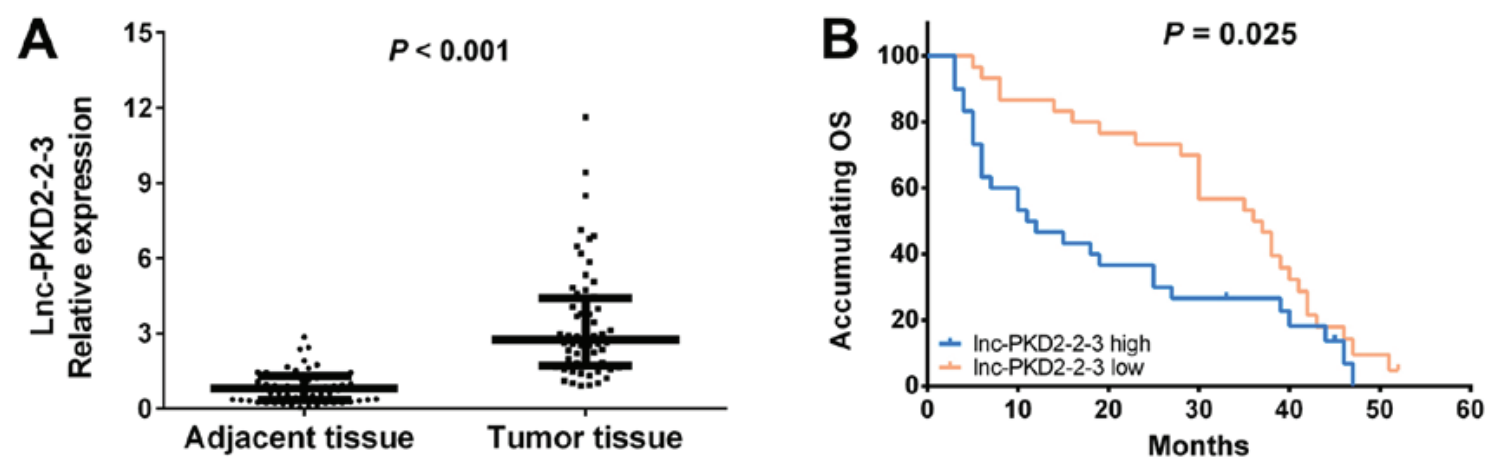

Figure 2. Correlation of lnc-PKD2-2-3 expression with prognosis. (A) Lnc-PKD2-2-3 levels were detected in CCA tumor tissues and in paired normal adjacent tissues. (B) Survival analysis of patients with high or low lnc-PKD2-2-3 expression. Comparison of expression was determined by Wilcoxon signed rank sum test. Survival analysis was performed with Kaplan-Meier curve followed by log-rank test. CCA, cholangiocarcinoma; OS, overall survival.
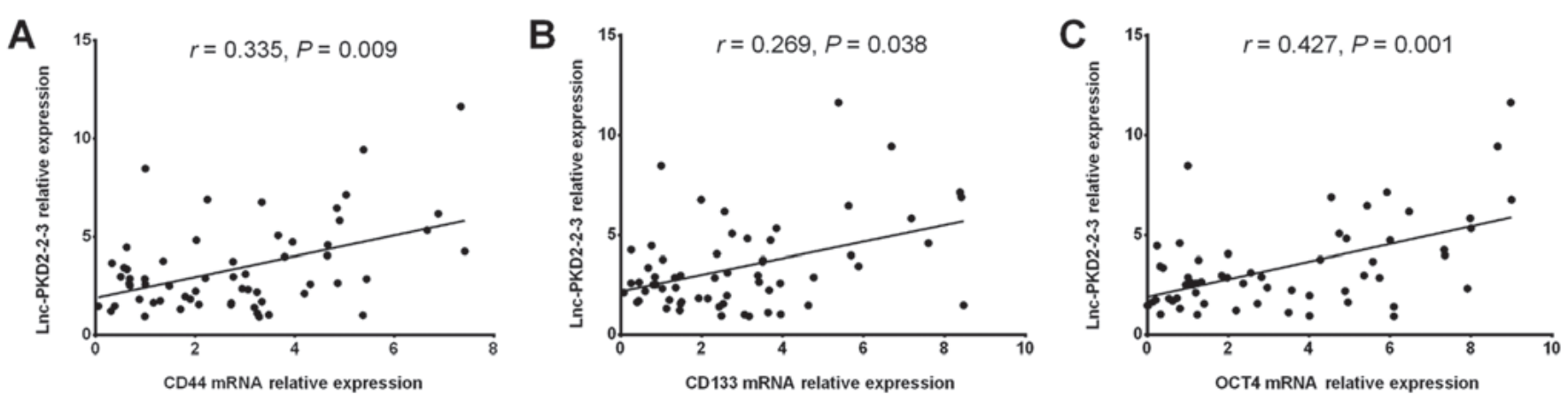

Figure 3. Correlation of lnc-PKD2-2-3 with CSC marker expression. Lnc-PKD2-2-3 expression was positively associated with (A) CD44, (B) CD133 and (C) OCT4 expression in cholangiocarcinoma tumor tissues. Correlation was determined by Spearman test. CSC, cancer stem cell; OCT4, octamer-binding transcription factor 4 .

using Pearson correlation coefficient and DAVID $(21,22)$ analyses, revealed that lnc-PKD2-2-3 was correlated with several oncogenes and stemness-associated pathways, including DGAT2, GPAM, HEPACAM, ATP1A1, PAFAH1B3, LOC728342, STAB2, SH2D3A and GTF2I. Therefore, its expression was further validated in 60 pairs of CCA tumor tissue and adjacent tissue by RT-qPCR. The results indicated that lnc-PKD2-2-3 levels were significantly increased in CCA tumor tissue compared with paired adjacent tissue (Fig. 2A). In addition, lnc-PKD2-2-3 levels were significantly associated with a higher ECOG performance score, poor differentiation, increased TNM stage and abnormal CEA levels, while it was not associated with age, sex, smoking, drinking, HBV infection, tumor site, tumor size, number of tumors, CA199 levels or surgery type (Table III).

All patients were followed up and 54 cases died, among which $48(89 \%)$ died due to tumor progression, 2 died from infection, 2 died from cardiovascular and cerebrovascular diseases and 2 died from other causes. The patients were divided into lnc-PKD2-2-3-high expression and lnc-PKD2-2-3-low expression groups, according to the median value of lnc-PKD2-2-3 levels in the tumor tissues. Survival analysis revealed that high expression of lnc-PKD2-2-3 was associated with a worse OS compared with subjects with low expression (Fig. 2B).

Correlation of lnc-PKD2-2-3 expression with CSC marker expression in CCA tissue. The expression of common CSC markers in the CCA tumor tissues was then measured, and it was revealed that lnc-PKD2-2-3 expression was positively correlated with CD44 (Fig. 3A), CD133 (Fig. 3B) and OCT4 (Fig. 3C) expression. These results implied that lnc-PKD2-2-3 expression may correlate with CCA stemness and may have the potential to serve as a CSC marker.

Inc-PKD2-2-3 expression of CCA cell lines post-transduction. lnc-PKD2-2-3 expression was increased in the overexpressing LV-Lnc group compared with the LV-NC group, while it was decreased in the silencing LVU6-Lnc group compared with the LVU6-NC group, following transduction with the respective lentivirus in TFK-1 cells (Fig. 4A) and Huh-28 cells (Fig. 4B). These results indicated that the expression of lnc-PKD2-2-3 was successfully upregulated or suppressed by the overexpressing or the silencing lentivirus, respectively.

lnc-PKD2-2-3 increases CSC marker expression and $C D 44^{+} \mathrm{CD} 133^{+}$cell proportion in CCA cell lines. The mRNA expression levels of CD44, CD133 and OCT4 were elevated in the LV-Lnc group compared with the LV-NC group, while it was reduced in the LVU6-Lnc group compared with the LVU6-NC group, in TFK-1 cells (Fig. 5A-C) and Huh-28 cells (Fig. 5G-I). The protein expression levels of these markers were also increased in the LV-Lnc group compared with the LV-NC group, and reduced in the LVU6-Lnc group compared with the LVU6-NC group, in TFK-1 cells (Fig. 5D) and Huh-28 cells (Fig. 5J). Notably, the $\mathrm{CD} 44^{+} \mathrm{CD} 133^{+}$cell proportion was increased in the LV-Lnc group compared with the LV-NC 
Table III. Patient characteristics.

\begin{tabular}{|c|c|c|c|}
\hline Parameters & CCA patients $(n=60)$ & lnc-PKD2-2-3 expression & P-value \\
\hline Age, n (\%) & & & 0.412 \\
\hline$\geq 60$ years & $26(43.3)$ & $2.449(1.629-3.829)$ & \\
\hline$<60$ years & $34(56.7)$ & $2.888(1.803-4.624)$ & \\
\hline Sex, n $(\%)$ & & & 0.358 \\
\hline Male & $42(70.0)$ & $2.760(1.911-4.624)$ & \\
\hline Female & $18(30.0)$ & $2.495(1.595-3.809)$ & \\
\hline Smoke, n (\%) & & & 0.404 \\
\hline Yes & $19(31.7)$ & $3.371(1.836-4.060)$ & \\
\hline No & $41(68.3)$ & $2.600(1.642-4.602)$ & \\
\hline Drink, n (\%) & & & 0.663 \\
\hline Yes & $29(48.3)$ & $2.636(1.642-4.160)$ & \\
\hline No & $31(51.7)$ & $2.887(1.751-4.739)$ & \\
\hline HBV infection, n (\%) & & & 0.094 \\
\hline Yes & $24(40.0)$ & $2.981(2.310-4.631)$ & \\
\hline No & $36(60.0)$ & $2.449(1.501-4.414)$ & \\
\hline ECOG performance score, n (\%) & & & 0.005 \\
\hline $1-2$ & $24(40.0)$ & $4.121(2.535-6.313)$ & \\
\hline 0 & $36(60.0)$ & $2.348(1.579-3.272)$ & \\
\hline Differentiation, n (\%) & & & 0.002 \\
\hline Poor & $26(43.3)$ & $3.244(2.598-5.924)$ & \\
\hline Well and moderate & $34(56.7)$ & $2.180(1.459-3.757)$ & \\
\hline Tumor site, n (\%) & & & 0.874 \\
\hline Intrahepatic & $18(30.0)$ & $2.563(1.609-4.230)$ & \\
\hline Perihilar & $21(35.0)$ & $2.887(1.895-4.525)$ & \\
\hline Extrahepatic & $21(35.0)$ & $2.864(1.667-4.530)$ & \\
\hline Tumor size, n (\%) & & & 0.052 \\
\hline$\geq 5 \mathrm{~cm}$ & $27(45.0)$ & $3.678(1.836-5.337)$ & \\
\hline$<5 \mathrm{~cm}$ & $33(55.0)$ & $2.517(1.677-3.409)$ & \\
\hline Tumor number, n (\%) & & & 0.172 \\
\hline Multiple & $20(33.3)$ & $3.378(1.799-5.646)$ & \\
\hline Single & $40(66.7)$ & $2.563(1.714-3.732)$ & \\
\hline T stage, n (\%) & & & 0.051 \\
\hline $\mathrm{T} 3 / \mathrm{T} 4$ & $17(28.3)$ & $3.750(2.445-6.686)$ & \\
\hline $\mathrm{T} 1 / \mathrm{T} 2$ & $43(71.7)$ & $2.600(1.633-3.987)$ & \\
\hline $\mathrm{N}$ stage, $\mathrm{n}(\%)$ & & & 0.002 \\
\hline N1 & $23(38.3)$ & $4.465(2.657-6.471)$ & \\
\hline No & $37(61.7)$ & $2.324(1.597-3.176)$ & \\
\hline TNM stage, n (\%) & & & 0.004 \\
\hline III/IV & $25(41.7)$ & 3.777 (2.553-6.154) & \\
\hline $\mathrm{I} / \mathrm{II}$ & $35(58.3)$ & $2.324(1.562-3.371)$ & \\
\hline CEA abnormal, n (\%) & & & 0.024 \\
\hline Yes $(\geq 5 \mathrm{ng} / \mathrm{ml})$ & $23(38.3)$ & $3.050(2.237-5.712)$ & \\
\hline No $(<5$ ng/ml $)$ & $37(61.7)$ & $2.282(1.597-3.770)$ & \\
\hline CA199 abnormal, n (\%) & & & 0.452 \\
\hline Yes $(\geq 37 \mathrm{U} / \mathrm{ml})$ & $32(53.3)$ & $3.447(1.633-5.073)$ & \\
\hline No $(<37 \mathrm{U} / \mathrm{ml})$ & $28(46.7)$ & $2.589(1.794-4.019)$ & \\
\hline Surgery, n (\%) & & & 0.207 \\
\hline LR & $17(28.3)$ & $2.982(2.558-4.441)$ & \\
\hline BT & $34(56.7)$ & $2.306(1.593-4.055)$ & \\
\hline WR & $9(15.0)$ & $3.117(1.840-5.772)$ & \\
\hline
\end{tabular}

CCA, cholangiocarcinoma; lnc-PDK2-2-3, long non-coding RNA PKD2-2-3; HBV, hepatitis B virus; ECOG, Eastern Cooperative Oncology Group; CEA, carcinoembryonic antigen; CA199, carbohydrate antigen199; LR, liver resection; BT, biliary duct resection; WR, Whipple resection. 

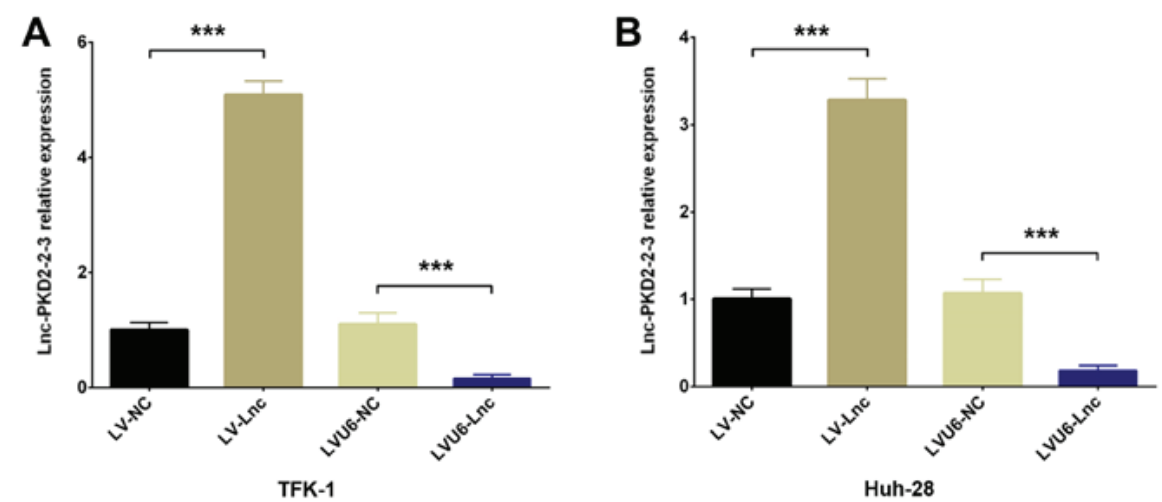

Figure 4. Lnc-PKD2-2-3 expression following lentivirus transduction. (A) TFK-1 cells and (B) Huh-28 cells were transduced with either lnc-PKD2-2-3-overexpressing lentivirus (LV-Lnc) and control (LV-NC), or with lnc-PKD2-2-3-silencing lentivirus (LVU6-Lnc) and control (LVU6-NC). ${ }^{* * *} \mathrm{P}<0.001$, with comparisons indicated by brackets. NC, negative control.
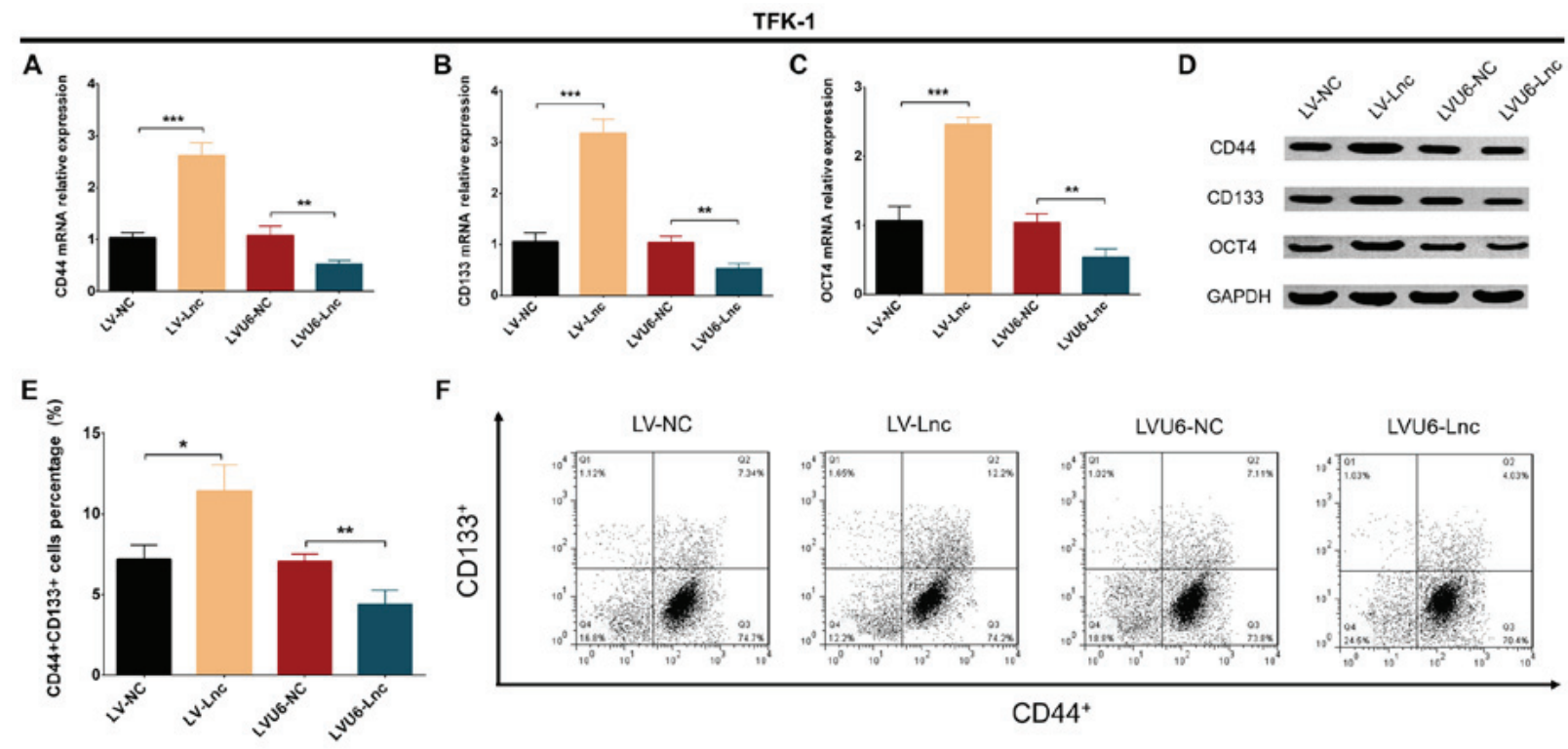

$\mathbf{F}$

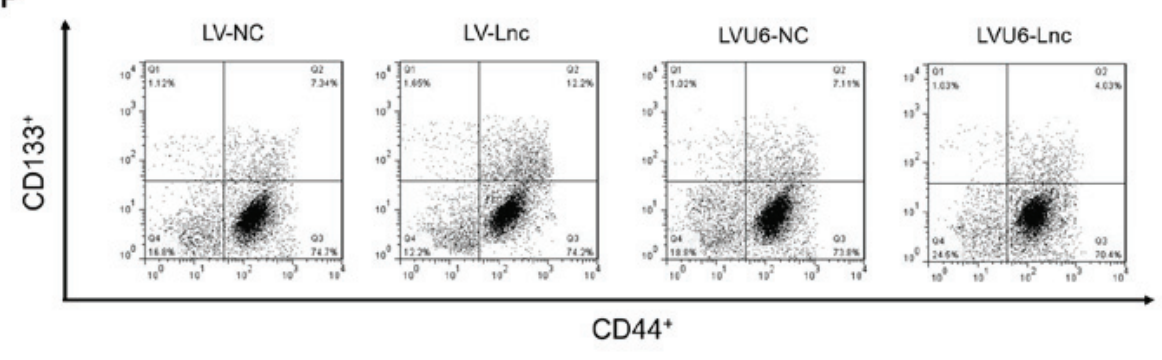

Huh-28

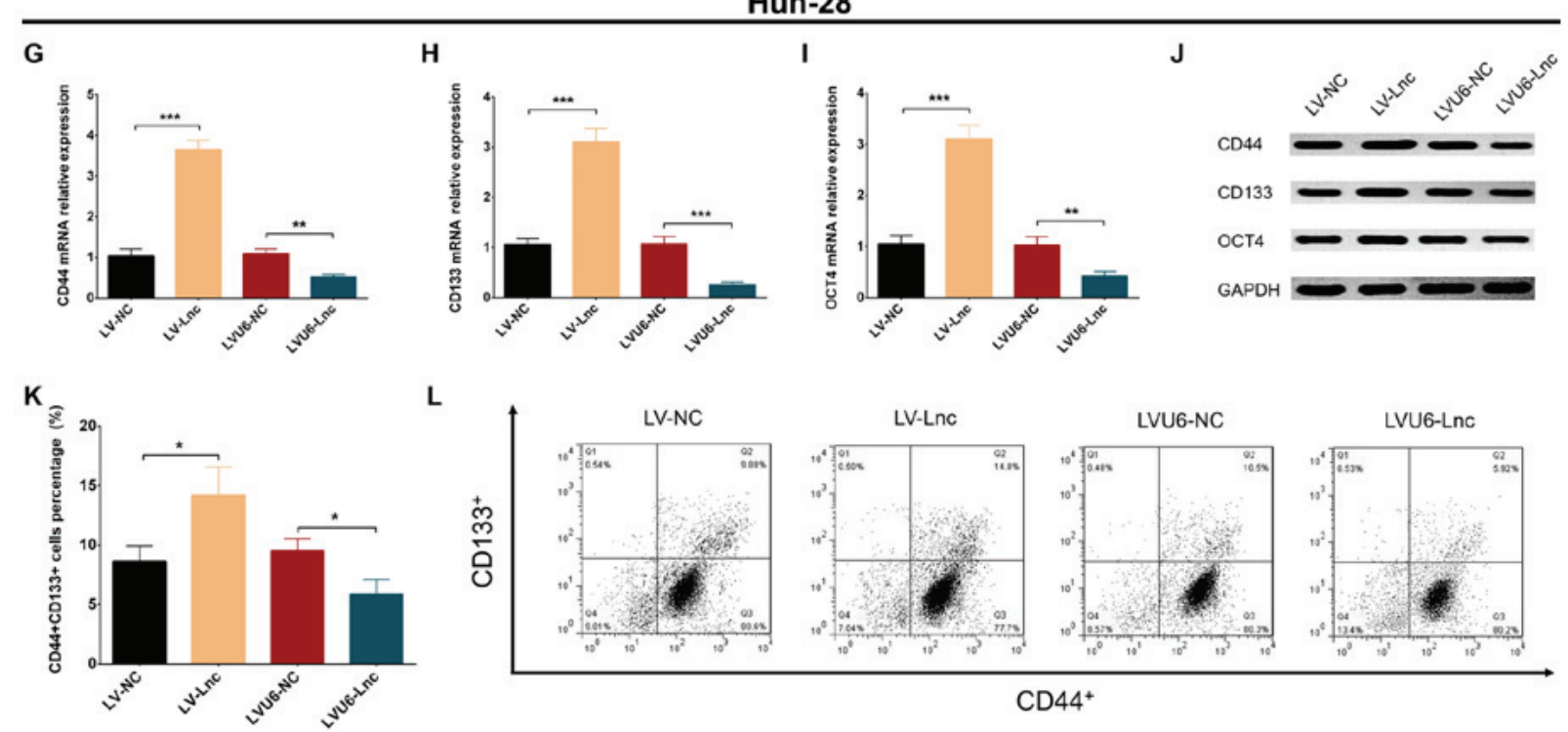

Figure 5. CSC marker expression and $\mathrm{CD} 44^{+} \mathrm{CD} 133^{+}$cell proportion following lentivirus transduction. (A) mRNA expression levels of CD44, (B) CD133 and (C) OCT4 in TFK-1 experimental cell groups. (D) Representative western blot of CSC marker expression in TFK-1 experimental cell groups. (E) Quantification and (F) representative plots of flow cytometry analysis for $\mathrm{CD} 44^{+} \mathrm{CD} 133^{+}$proportions in TFK-1 experimental cell groups. (G) mRNA expression levels of CD44, (H) CD133 and (I) OCT4 in Huh-28 experimental cell groups. (J) Representative western blot of CSC marker expression in Huh-28 experimental cell groups. (K) Quantification and (L) representative plots of flow cytometry analysis for $\mathrm{CD} 44^{+} \mathrm{CD} 133^{+}$proportions in Huh- 28 experimental cell groups. "P<0.05, ${ }^{* *} \mathrm{P}<0.01$ and ${ }^{* * *} \mathrm{P}<0.001$, with comparisons indicated by brackets. CSC, cancer stem cell; NC, negative control. 


\section{TFK-1}

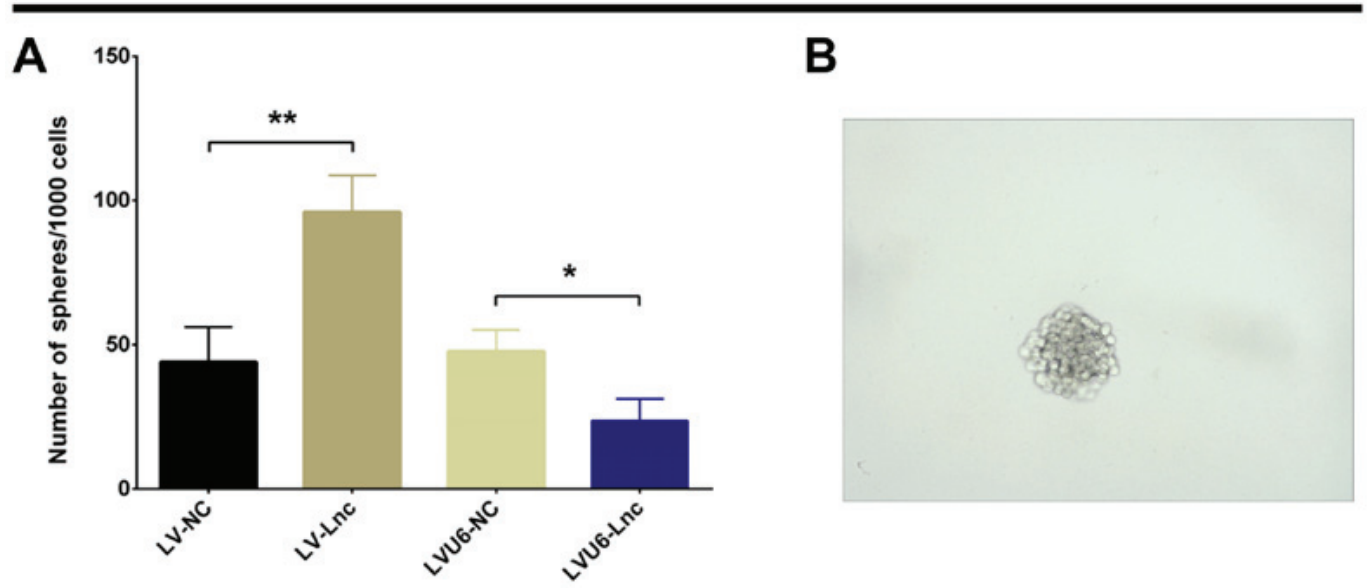

Huh-28

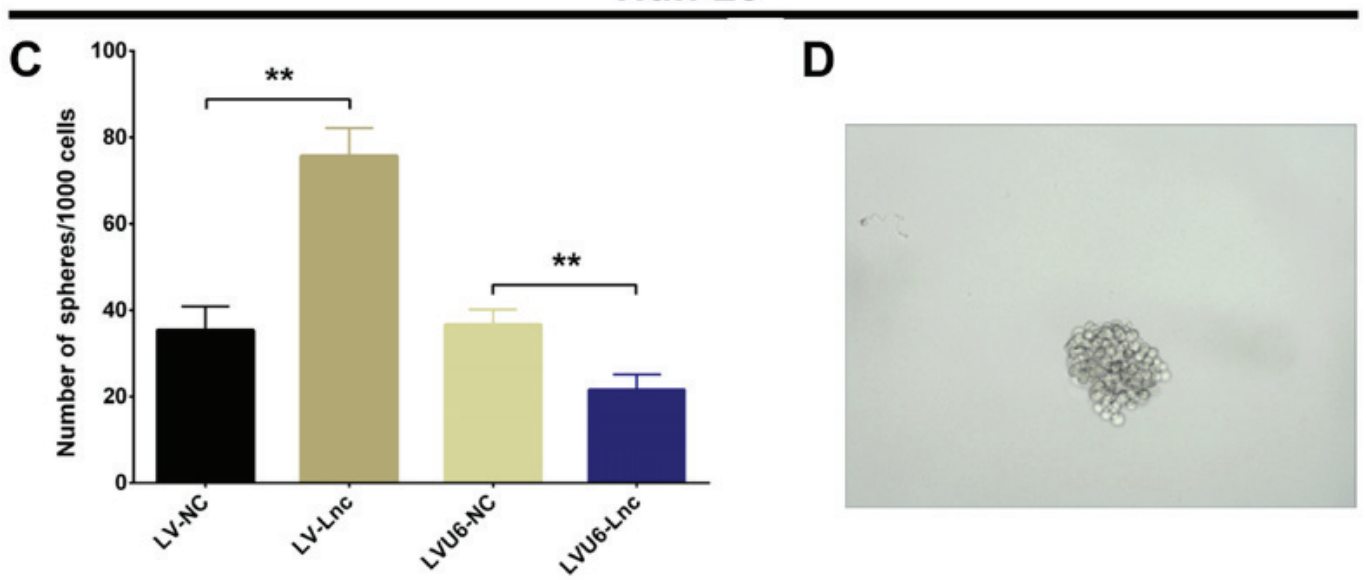

Figure 6. Sphere formation efficiency following lentivirus transduction. (A) Quantification and (B) representative microscopy image (magnification, x200) from sphere formation assay in TFK-1 experimental cell groups. (C) Quantification and (D) representative microscopy image (magnification, x200) from sphere formation assay in Huh-28 experimental cell groups. ${ }^{*} \mathrm{P}<0.05$ and ${ }^{* *} \mathrm{P}<0.01$, with comparisons indicated by brackets. NC, negative control.

group, and decreased in the LVU6-Lnc group compared with the LVU6-NC group, in TFK-1 cells (Fig. 5E and F) and Huh-28 cells (Fig. $5 \mathrm{~K}$ and L). These results implied that lnc-PKD2-2-3 overexpression may increase CCA stemness.

lnc-PKD2-2-3 enhances sphere formation efficiency in CCA cell lines. The sphere formation assay revealed that the sphere formation efficiency was increased in the LV-Lnc group compared with the LV-NC group, while it was reduced in the LVU6-Lnc group compared with the LVU6-NC group, in TFK-1 cells (Fig. 6A and B) and in Huh-28 cells (Fig. 6C and D). These results further suggested that lnc-PKD2-2-3 overexpression increased CCA stemness.

lnc-PKD2-2-3 increases drug resistance to 5-FU in CCA cell lines. Following 5-FU treatment, the viability of TFK-1 cells in the LV-Lnc +5 -FU group was increased compared with the LV-NC + 5-FU group, while the viability in the LVU6-Lnc + 5-FU group was decreased compared with the LVU6-NC + 5-FU group (Fig. 7A). By contrast, the apoptotic rate of TFK-1 cells was repressed in the LV-Lnc $+5-\mathrm{FU}$ group compared with the LV-NC + 5-FU group, while it was enhanced in the LVU6-Lnc + 5-FU group compared with the LVU6-NC + 5-FU group (Fig. 7B and D). Furthermore, in
TFK-1 cells, the apoptotic marker C-caspase- 3 was decreased in the LV-Lnc + 5-FU group compared with the LV-NC + 5-FU group, while it was increased in the LVU6-Lnc + 5-FU group compared with the LVU6-NC + 5-FU group (Fig. 7C). The opposite trend was observed for the anti-apoptotic marker Bcl-2 (Fig. 7C). Similar to the TFK-1 cell lines, experiments in the Huh-28 cell line also indicated that the cell viability was enhanced (Fig. 7E), while cell apoptosis was inhibited (Fig. 7F-H) by lnc-PKD2-2-3 in the presence of 5-FU treatment. These results demonstrated that lnc-PKD2-2-3 overexpression increased drug resistance to 5-FU in CCA cell lines, which further suggested that 1nc-PKD2-2-3 may have the potential to increase CCA stemness.

lnc-PKD2-2-3 levels are increased in CCA stem-like cells. CCA stem-like cells were obtained by generation of 5-FU-resistant CCA cells (termed R-TFK-1 and R-Huh-28), that were further selected in a sphere formation assay, followed by isolation through centrifugation. Cell viability was similar between the R-TFK-1 cells treated with 5-FU and the untreated R-TFK-1 cells (Fig. 8A), as well as between the R-Huh-28 cells treated with 5-FU and the untreated R-Huh-28 cells (Fig. 8D), confirming that the generated R-TFK-1 and R-Huh-28 cells were resistant to 5-FU. Of note, R-TFK-1 cells 
TFK-1

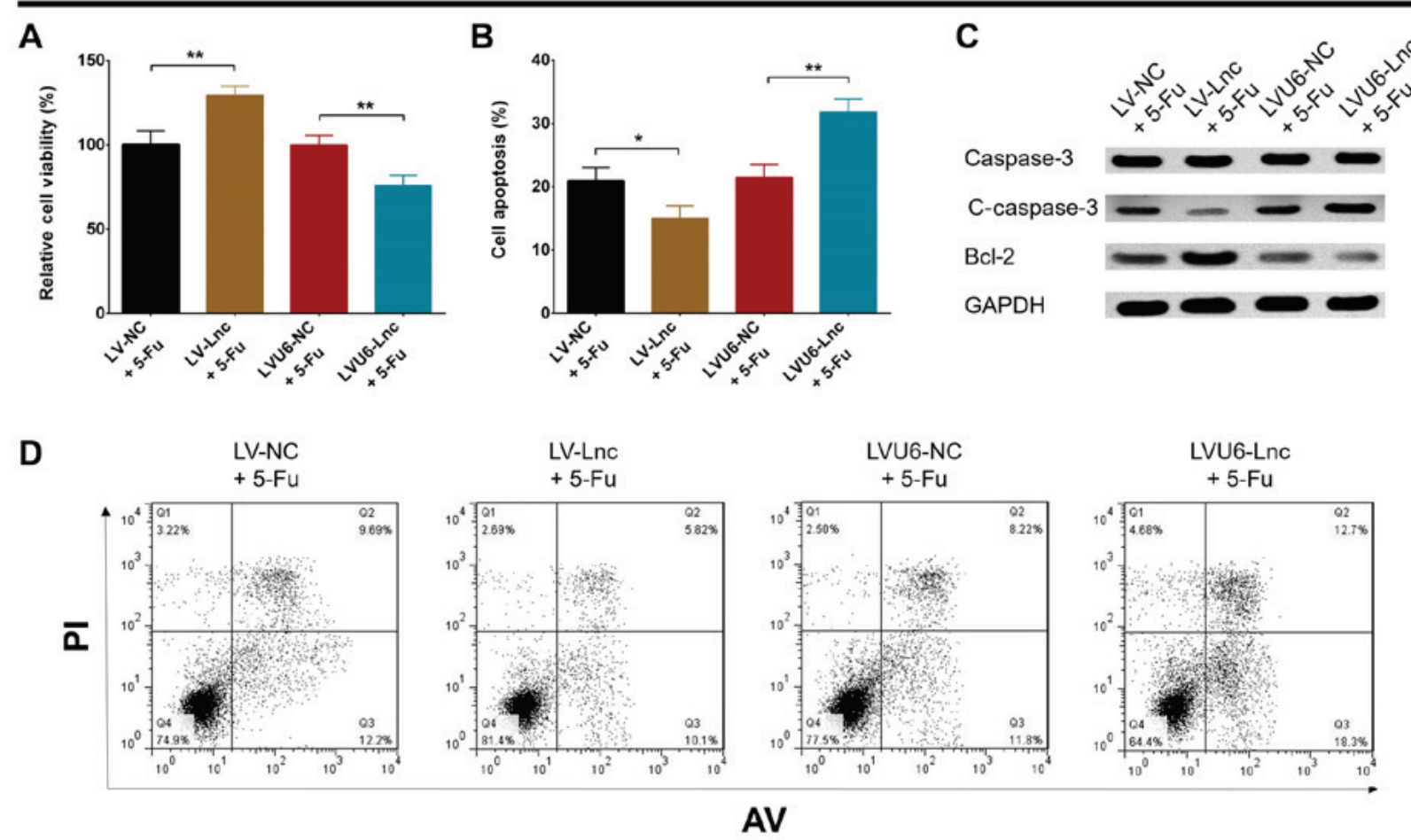

Huh-28
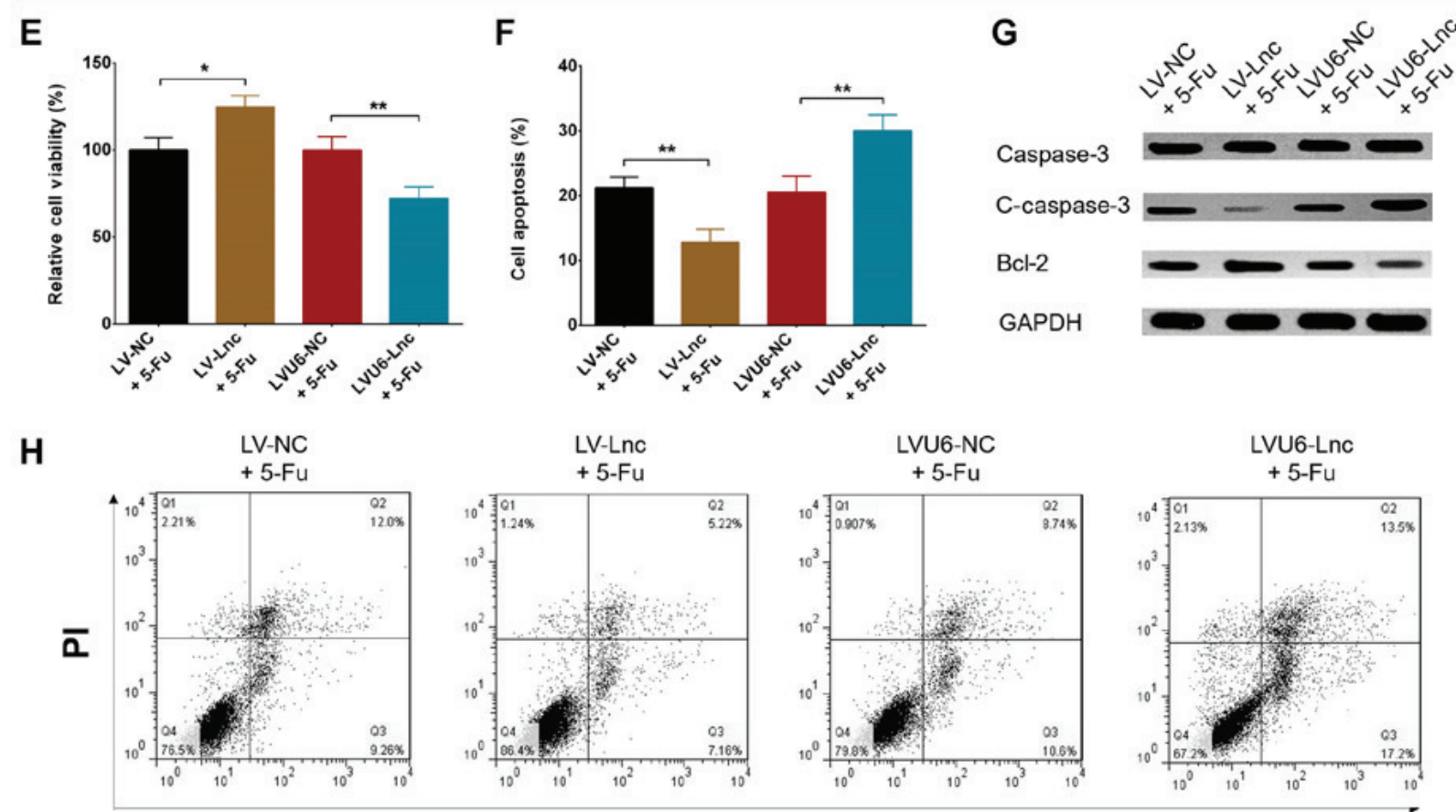

\section{AV}

Figure 7. Drug resistance to 5-FU following lentivirus transduction. (A) Cell viability was measured in TFK-1 experimental cell groups by CCK-8 assay (B) Cell apoptosis was measured in TFK-1 experimental cell groups by flow cytometry analysis. (C) Expression of apoptosis-related proteins was evaluated in TFK-1 experimental cell groups by western blotting. (D) Representative plots from flow cytometry analysis in panel B. (E) Cell viability was measured in Huh-28 experimental cell groups by CCK-8 assay. (F) Cell apoptosis was measured in Huh-28 experimental cell groups by flow cytometry analysis. (G) Expression of apoptosis-related proteins was evaluated in Huh-28 experimental cell groups by western blotting. (H) Representative plots from flow cytometry analysis in panel F. " $\mathrm{P}<0.05$ and ${ }^{* *} \mathrm{P}<0.01$, with comparisons indicated by brackets. 5-FU, 5-fluorouracil; CCK-8, cell counting kit-8; NC, negative control; Bcl-2, B-cell lymphoma-2; PI, propidium iodide; AV, Annexin V.

exhibited increased sphere formation efficiency compared with parental TFK-1 cells (Fig. 8B); a representative sphere is displayed in Fig. 8C. R-Huh-28 cells also exhibited increased sphere formation efficiency compared with the parental 
TFK-1
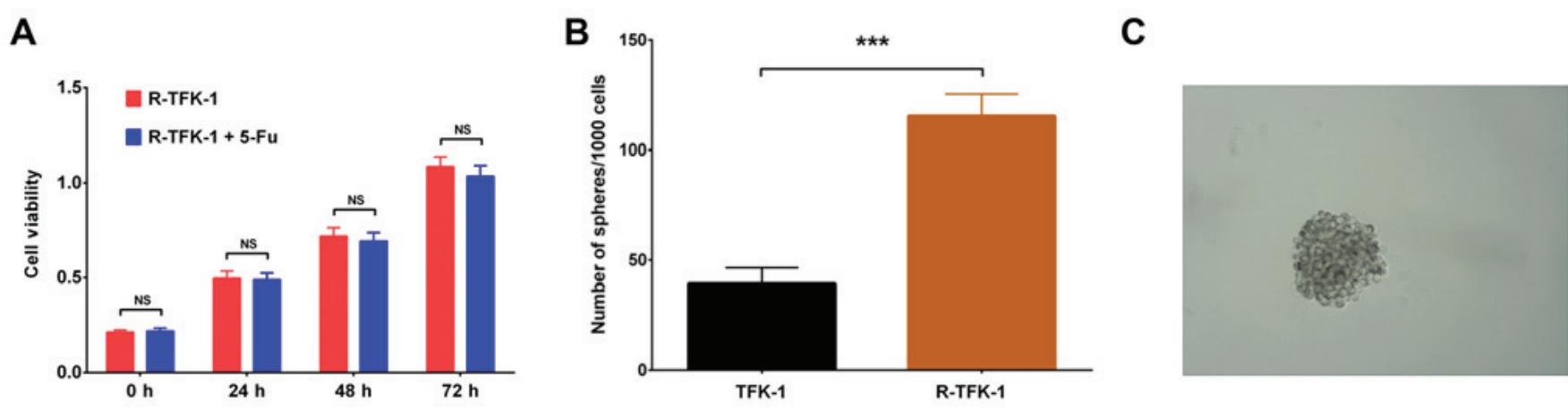

Huh-28
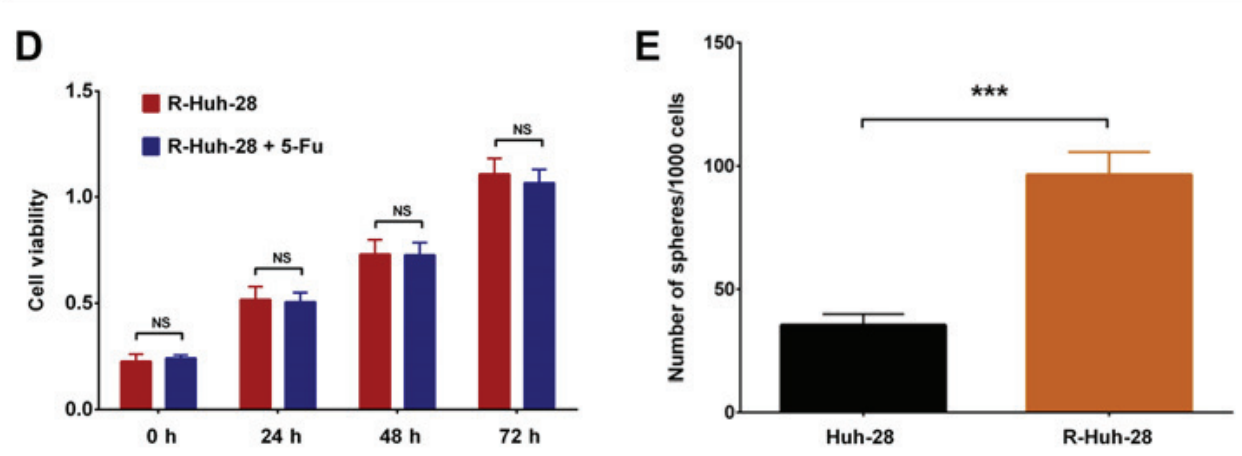

$\mathbf{F}$

Figure 8. Generation of CCA stem-like cells. CCA stem-like cells were constructed by 5-FU repeated treatment, sphere formation assay and sphere isolation. (A) Drug resistant R-TFK-1 cells were constructed and confirmed to have equal cell viability with or without 5-FU treatment. (B) Quantification and (C) representative image (magnification, x200) of sphere formation assay in R-TFK-1 and TFK-1 cells. (D) Similarly, drug resistant R-Huh-28 cells were constructed and confirmed to have equal cell viability with or without 5-FU treatment. (E) Quantification and (F) representative image (magnification, x200) of sphere formation assay in R-Huh-28 and Huh-28 cells. ${ }^{* * * *} \mathrm{P}<0.001$, with comparisons indicated by brackets. CCA, cholangiocarcinoma; 5-FU, 5-fluorouracil; $\mathrm{R}$, resistant; NS, not significant.

Huh-28 cells (Fig. 8E); a representative sphere is displayed in Fig. 8F.

Subsequently, CD44, CD133 and OCT4 expression was measured in spheres of TFK-1 cells (S-TFK-1) and parental TFK-1 cells, as well as in spheres of Huh-28 cells (S-Huh-28) and parental Huh-28 cells. The results revealed that mRNA and protein expression levels of CD44, CD133 and OCT4 were increased in S-TFK-1 cells compared with parental TFK-1 cells (Fig. 9A-D), and in S-Huh-28 cells compared with parental Huh-28 cells (Fig. 9F-I). These results implied the successful generation of CCA stem-like cells by sphere isolation (S-TFK-1 and S-Huh-28 groups). Finally, lnc-PKD2-2-3 expression was demonstrated to be significantly increased in S-TFK-1 cells compared with parental TFK-1 cells (Fig. 9E), and in S-Huh-28 cells compared with parental Huh-28 cells (Fig. 9J). These results further indicated that lnc-PKD2-2-3 may serve as a novel marker for CSCs in CCA.

\section{Discussion}

In the present study, lncRNA expression profiles in CCA were first obtained by microarray analysis, which provided 4,223 upregulated and 4,596 downregulated lncRNAs in CCA tumor tissues compared with paired adjacent tissues. These dysregulated lncRNAs were identified to be enriched in various cancer-associated pathways. Furthermore, lnc-PKD2-2-3, one of the most upregulated IncRNAs identified in the microarray analysis, was confirmed to be upregulated in CAA tissues. High lnc-PKD2-2-3 expression was determined to be associated with a higher ECOG performance score, poor differentiation, advanced TNM stage and increased CEA, and was a predictor of poor prognosis in CCA patients. Of note, it was also revealed that overexpression of lnc-PKD2-2-3 in CCA cell lines significantly increased CSC marker expression (CD44, CD133 and OCT4) and $\mathrm{CD}_{4}{ }^{+} \mathrm{CD} 133^{+}$cell proportion, enhanced sphere formation efficiency and increased drug resistance to 5-FU, indicating that lnc-PKD2-2-3 may promote CCA stemness. In addition, lnc-PKD2-2-3 was positively correlated with CSC markers in CCA tumor tissues and was markedly upregulated in CCA stem-like cells compared with normal CCA cells, suggesting that lnc-PKD2-2-3 may potentially serve as a marker for CSCs in CCA.

IncRNAs, as a group of newly discovered non-coding RNAs, participate in various molecular biological processes, and their differential expression patterns have been implicated in the pathogenesis of numerous diseases, including vascular disease, diabetes and various types of cancer (24-26). A previous study identified 10,680 dysregulated lncRNAs in colorectal cancer tissues compared with paired adjacent normal tissues by microarray analysis, and 2,970 lncRNAs were differentially expressed in tumor tissues with liver metastasis compared with tumor tissues without liver metastasis, indicating that lncRNA profiles are involved in carcinogenesis and metastasis of colorectal cancer (27). Another two studies 
TFK-1
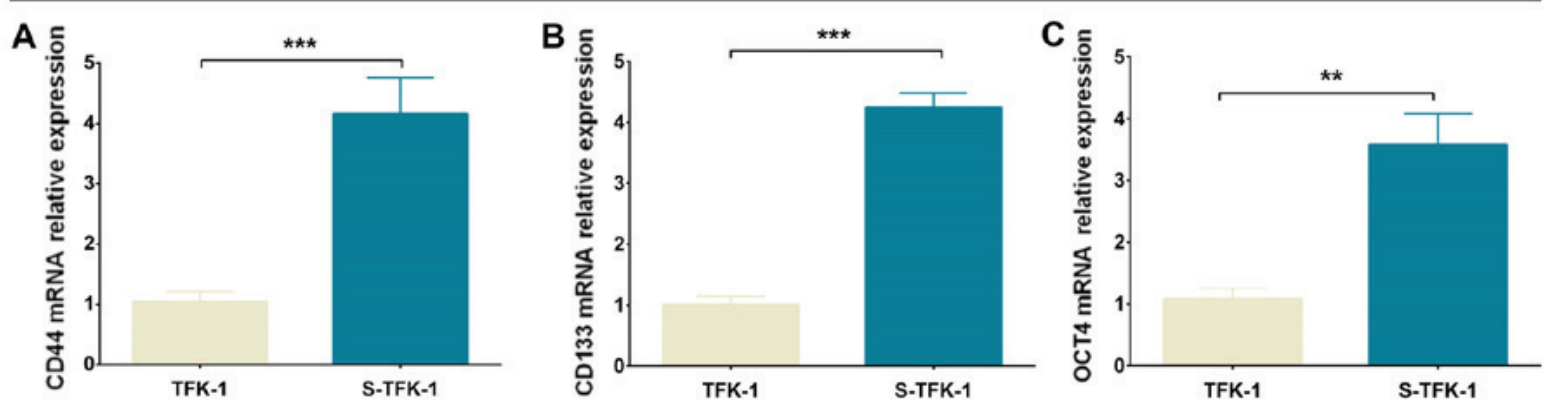

D

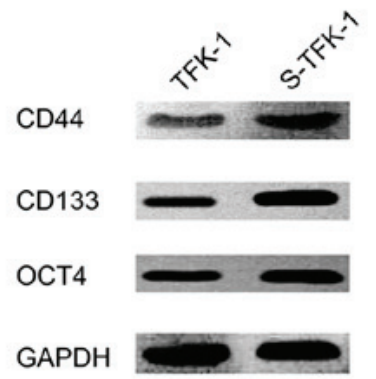

E

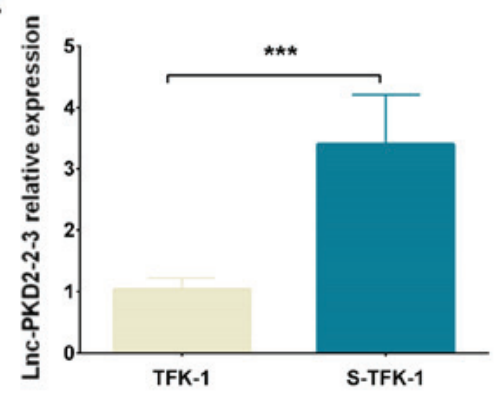

Huh-28
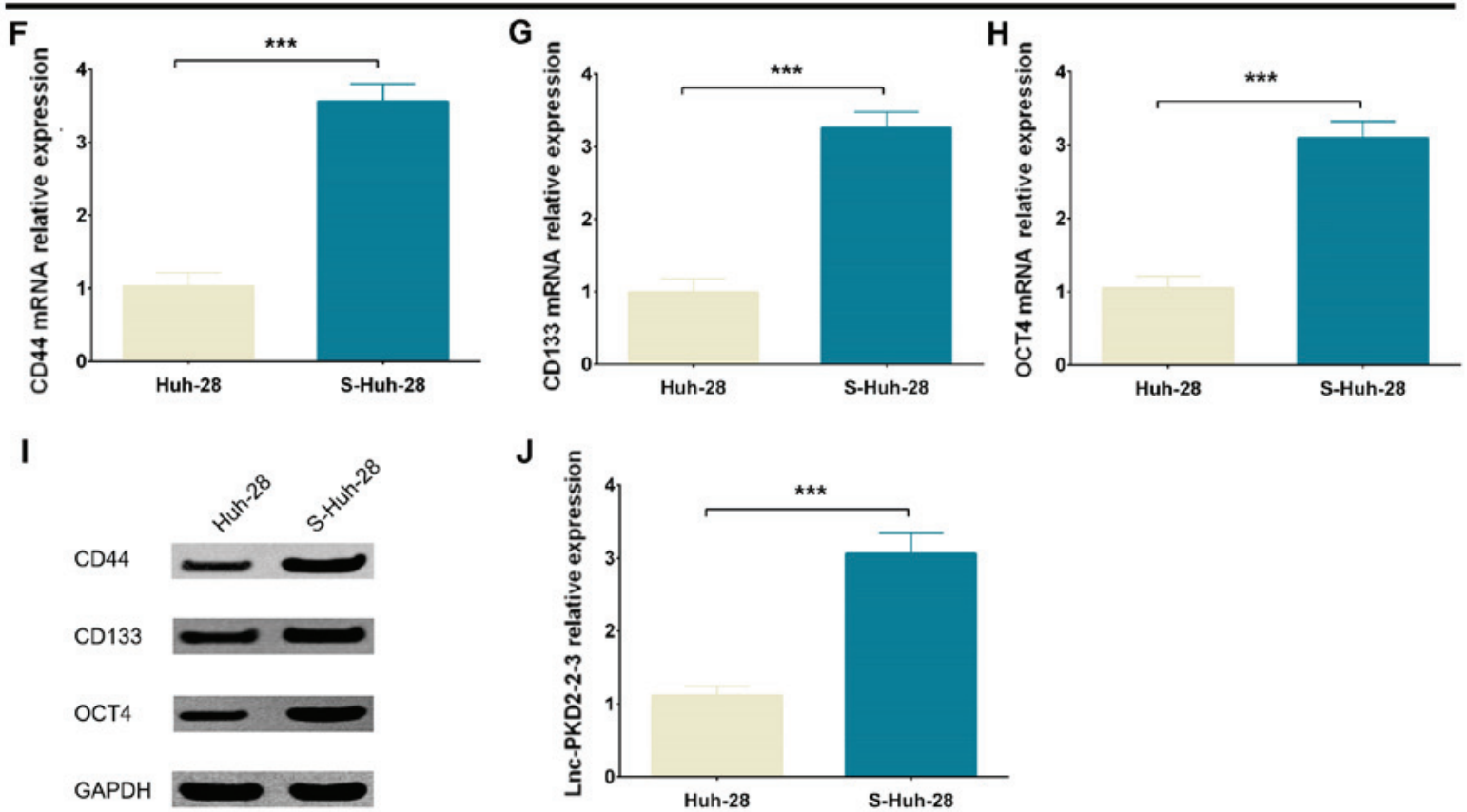

Figure 9. Validation of cholangiocarcinoma stem-like cells and lnc-PKD2-2-3 expression. (A) mRNA levels of CD44, (B) CD133 and (C) OCT4, as well as (D) their protein levels were measured in the S-TFK-1 and parental TFK-1 cells. (E) Lnc-PKD2-2-3 levels in the S-TFK-1 and parental TFK-1 cells. (F) mRNA levels of CD44, (G) CD133 and (H) OCT4, as well as (I) their protein levels were measured in the S-Huh-28 and parental Huh-28 cells. (J) Lnc-PKD2-2-3 levels in the S-Huh-28and parental Huh-28 cells. ${ }^{* *} \mathrm{P}<0.01$ and ${ }^{* * *} \mathrm{P}<0.001$, with comparisons indicated by brackets. S, stem-like; OCT4, octamer-binding transcription factor 4.

reported 214 dysregulated IncRNAs in hepatocellular carcinoma tissues compared with paired adjacent tissues by high-throughput RNA sequencing and 234 aberrant lncRNAs were identified in the blood of hepatocellular carcinoma patients with vs. without lymph node metastasis $(28,29)$. As for CCA, only one previous original study explored the lncRNA expression patterns between extrahepatic CCA tissue and normal non-cancerous tissue using microarray, and it only used a limited set of probes for lncRNA detection; thus, only
268 dysregulated lncRNAs were identified and most aberrant lncRNAs remained undetected (30). In the present study, $>80,000$ probes were designed in a microarray and a total of 46,846 lncRNAs were detected in $>50 \%$ of samples of CCA tumor tissue and adjacent tissue, which were included in the bioinformatics analysis. A total of 4,223 upregulated lncRNAs and 4,596 downregulated lncRNAs were identified in CCA tumor tissues compared with paired adjacent tissues, and these dysregulated lncRNAs were enriched in cancer-associated 
pathways, including ECM-receptor interaction, alcoholism and PI3K/Akt signaling. These results indicated that dysregulated lncRNA profiles may have critical roles in CCA carcinogenesis.

lncRNAs have also been reported to serve as markers of tumor development, progression and prognosis in numerous types of cancer. For instance, lncRNA TP73-AS1 was reported to be upregulated in tumor tissue and to be associated with larger tumor size, TNM stage and shorter OS in gastric cancer patients (31). IncRNA serine peptidase inhibitor, Kunitz type 1-AS1 was determined to be overexpressed in cancer tissue and to be associated with a higher risk of regional lymph node metastasis and distant metastasis, and it is also an independent predictor of shorter relapse-free survival in colorectal cancer patients (32). In addition, upregulated lncRNA gastric carcinoma proliferation enhancing transcript 1 was reported to be associated with vascular invasion, cirrhosis, tumor size and Edmondson-Steiner grade, and is a predictor of poor survival (33). In the present study, since lnc-PKD2-2-3 was one of the most upregulated lncRNAs detected by microarray, and bioinformatics analysis revealed that it was associated with several oncogenes and stemness-associated genes, its expression was further validated in 60 pairs of CCA tumor tissue and adjacent tissue using RT-qPCR. It was observed that lnc-PKD2-2-3 was upregulated and associated with a higher ECOG performance score, poor differentiation, advanced TNM stage and increased CEA, as well as associated with poor prognosis in CCA patients.

Of note, the present study was the first to report on the function of lnc-PKD2-2-3 in carcinogenesis. The roles of lnc-PKD2-2-3 in cancer may be based on the following mechanisms: i) lnc-PKD2-2-3 promotes CCA stemness and then stimulates tumor progression and enhances drug resistance, thus leading to tumor progression and poor prognosis, which was validated in the in vitro experiments of the present study; ii) lnc-PKD2-2-3 may increase CCA progression via sponging tumor suppressive target miRNAs or enhancing the function of oncogenes or cancer-associated pathways, thus giving rise to advanced tumor features and unfavorable prognosis. The latter hypothesis requires further investigation. The results of the present study indicated that lnc-PKD2-2-3 may serve as a marker of tumor development, progression and prognosis in CCA.

CSCs, a concept first put forward a decade ago and initially proposed in acute myeloid leukemia, are a small population of stem-like cancer cells exhibiting the capacity of self-renewal and differentiation into various types of cancer cells, which are considered to have a crucial role in increasing drug resistance and disease relapse in various types of cancer $(34,35)$. In experimental models, cytotoxic agents have been demonstrated to lack efficacy in killing CSCs, and CSCs are also reported to be responsible for the metastasis and re-growth of tumors after unsuccessful treatment $(34,35)$. Thus, deeper investigation of underlying mechanisms and additional targets/markers of CSCs are of critical importance for developing treatments for cancer, including CCA. Recently, a small number of unique IncRNAs have been observed to participate in the regulation of cancer-cell stemness and to have potential as markers for CSCs in several cancer types. For instance, lnc-THOR was reported to increase gastric cancer cell stemness by upregulating CSC marker expression and the capacity of spheroid formation of cells via enhancing the stability of SOX9 mRNA (13). lncRNA transcription factor 7 has been reported to induce CSC self-renewal (detected by CSC marker expression and spheroid formation efficiency) and tumor propagation via regulation of Wnt signaling pathways (14). Furthermore, inhibition of lncRNA FEZF1-AS1 was demonstrated to decrease breast cancer stem cell stemness by measurement of CSC marker expression and determination of the mammosphere-forming ability, through targeting the miR-30a and NANOG axis (15). As for CCA, no previous study has reported on the role of lncRNAs in stemness regulation. To the best of our knowledge, the present study is the first to report that lnc-PKD2-2-3 increased CSC marker expression and $\mathrm{CD} 44^{+} \mathrm{CD} 133^{+}$cell proportion, improved the sphere formation efficiency and enhanced drug resistance to 5-FU in CCA cell lines, indicating that lnc-PKD2-2-3 may promote CCA stemness. Furthermore, lnc-PKD2-2-3 was positively correlated with CSC markers in CCA tumor tissues and was markedly upregulated in CCA stem-like cells compared with normal CCA cells, implying that lnc-PKD2-2-3 may potentially serve as a marker for CSCs in CCA. Notably, the CD133 expression in the control group of TFK-1 and Huh-28 was numerically higher than that reported in a previous study (36), while it was similar compared with that in other previous studies $(37,38)$; this discrepancy may result from different detection methods, exposure time of western blot, dilution of antibody and experimental conditions among the different studies. Considering the aforementioned data, the present findings would provide novel evidence for application of lncRNA as a target to eliminate CSCs, and to further decrease treatment refraction and disease relapse in CCA.

In conclusion, lnc-PKD2-2-3, identified from lncRNA expression profiling, was associated with pejorative tumor features and poor prognosis, and may serve as a CSC marker that is associated with CD44, CD133 and OCT4 expression, and $\mathrm{CD} 44^{+} \mathrm{CD} 133^{+}$cell proportion. In addition, lnc-PKD2-2-3 overexpression increased sphere formation efficiency and enhanced drug resistance to 5-FU in CCA cell lines. The present study provided novel evidence for the application of a lncRNA as a target to eliminate CSCs and to reverse drug resistance and disease relapse in patients with CCA.

\section{Acknowledgements}

Not applicable.

\section{Funding}

No funding was received.

\section{Availability of data and materials}

All data generated or analysed during this study are included in this published article.

\section{Authors' contributions}

$\mathrm{ZZ}$ contributed to the concept and design of the study and editing of the manuscript. GQ provided the study materials and revised the manuscript. DM, FL and DS are responsible 
for the data analysis and manuscript writing. All authors read and approved the final manuscript.

\section{Ethics approval and consent to participate}

The present study was approved by the Ethics Committee of the Second Affiliated Hospital of Harbin Medical University (Harbin, China) and all patients provided written informed consent prior to enrollment.

\section{Patient consent for publication}

Not applicable.

\section{Competing interests}

The authors declare that they have no competing interests.

\section{References}

1. Shin HR, Oh JK, Masuyer E, Curado MP, Bouvard V, Fang YY, Wiangnon S, Sripa B and Hong ST: Epidemiology of cholangiocarcinoma: An update focusing on risk factors. Cancer Sci 101: 579-585, 2010.

2. Mosadeghi S, Liu B, Bhuket T and Wong RJ: Sex-specific and race/ethnicity-specific disparities in cholangiocarcinoma incidence and prevalence in the USA: An updated analysis of the 2000-2011 Surveillance, Epidemiology and End Results registry. Hepatol Res 46: 669-677, 2016.

3. Razumilava N and Gores GJ: Cholangiocarcinoma. Lancet 383: $2168-2179,2014$

4. Bray F, Ferlay J, Soerjomataram I, Siegel RL, Torre LA and Jemal A: Global cancer statistics 2018: GLOBOCAN estimates of incidence and mortality worldwide for 36 cancers in 185 countries. CA Cancer J Clin 68: 394-424, 2018.

5. Rizvi S, Khan SA, Hallemeier CL, Kelley RK and Gores GJ: Cholangiocarcinoma - evolving concepts and therapeutic strategies. Nat Rev Clin Oncol 15: 95-111, 2018.

6. Bridgewater J, Galle PR, Khan SA, Llovet JM, Park JW, Patel T, Pawlik TM and Gores GJ: Guidelines for the diagnosis and management of intrahepatic cholangiocarcinoma. J Hepatol 60: $1268-1289,2014$

7. Rizvi S and Gores GJ: Emerging molecular therapeutic targets for cholangiocarcinoma. J Hepatol 67: 632-644, 2017.

8. Balas MM and Johnson AM: Exploring the mechanisms behind long noncoding RNAs and cancer. Noncoding RNA Res 3: 108-117, 2018.

9. Rafiee A, Riazi-Rad F, Havaskary M and Nuri F: Long noncoding RNAs: Regulation, function and cancer. Biotechnol Genet Eng Rev 34: 153-180, 2018.

10. Evans JR, Feng FY and Chinnaiyan AM: The bright side of dark matter: lncRNAs in cancer. J Clin Invest 126: 2775-2782, 2016.

11. Schmitt AM and Chang HY: Long noncoding RNAs in cancer pathways. Cancer Cell 29: 452-463, 2016.

12. Prensner JR and Chinnaiyan AM: The emergence of lncRNAs in cancer biology. Cancer Discov 1: 391-407, 2011.

13. Song H, Xu Y, Shi L, Xu T, Fan R, Cao M, Xu W and Song J: LncRNA THOR increases the stemness of gastric cancer cells via enhancing SOX9 mRNA stability. Biomed Pharmacother 108: 338-346, 2018.

14. Wang Y, He L, Du Y, Zhu P, Huang G, Luo J, Yan X, Ye B, Li C, Xia P, et al: The long noncoding RNA lncTCF7 promotes self-renewal of human liver cancer stem cells through activation of Wnt signaling. Cell Stem Cell 16: 413-425, 2015.

15. Zhang Z, Sun L, Zhang Y, Lu G, Li Y and Wei Z: Long non-coding RNA FEZF1-AS1 promotes breast cancer stemness and tumorigenesis via targeting miR-30a/Nanog axis. J Cell Physiol 233: 8630-8638, 2018.

16. Batlle E and Clevers H: Cancer stem cells revisited. Nat Med 23: 1124-1134, 2017.

17. Pützer BM, Solanki M and Herchenröder O: Advances in cancer stem cell targeting: How to strike the evil at its root. Adv Drug Deliv Rev 120: 89-107, 2017.
18. Li Y, Cai Q, Li W, Feng F and Yang L: Long non-coding RNA EPIC1 promotes cholangiocarcinoma cell growth. Biochem Biophys Res Commun 504: 654-659, 2018.

19. Guo L, Zhou Y, Chen Y, Sun H, Wang Y and Qu Y: LncRNA ASAP1-IT1 positively modulates the development of cholangiocarcinoma via hedgehog signaling pathway. Biomed Pharmacother 103: 167-173, 2018.

20. Li Z, Li J, Ji D, Leng K, Xu Y, Huang L, Jiang X and Cui Y: Overexpressed long noncoding RNA Sox2ot predicts poor prognosis for cholangiocarcinoma and promotes cell proliferation and invasion. Gene 645: 131-136, 2018.

21. Huang W, Sherman BT and Lempicki RA: Systematic and integrative analysis of large gene lists using DAVID bioinformatics resources. Nat Protoc 4: 44-57, 2009.

22. Huang W, Sherman BT and Lempicki RA: Bioinformatics enrichment tools: Paths toward the comprehensive functional analysis of large gene lists. Nucleic Acids Res 37: 1-13, 2009.

23. Livak KJ and Schmittgen TD: Analysis of relative gene expression data using real-time quantitative PCR and the 2(-Delta Delta $\mathrm{C}(\mathrm{T})$ ) method. Methods 25: 402-408, 2001.

24. Kumar S, Williams D, Sur S, Wang JY and Jo H: Role of flow-sensitive microRNAs and long noncoding RNAs in vascular dysfunction and atherosclerosis. Vascul Pharmacol 114: 76-92, 2019.

25. Feng SD, Yang JH, Yao CH, Yang SS, Zhu ZM, Wu D, Ling HY and Zhang L: Potential regulatory mechanisms of lncRNA in diabetes and its complications. Biochem Cell Biol 95: 361-367, 2017.

26. Quan J, Pan X, Zhao L, Li Z, Dai K, Yan F, Liu S, Ma H and Lai Y: LncRNA as a diagnostic and prognostic biomarker in bladder cancer: A systematic review and meta-analysis. OncoTargets Ther 11: 6415-6424, 2018.

27. Wang X, Liu F, Liu X, Wang F, Liao X, Chen Y, Mao Y, Hua D and Ge X: Long non-coding RNA expression profiles reveals AK098783 is a biomarker to predict poor prognosis in patients with colorectal cancer. Jpn J Clin Oncol 48: 480-484, 2018

28. Yao J, Wu L, Meng X, Yang H, Ni S, Wang Q, Zhou J, Zhang Q, Su K, Shao L, et al: Profiling, clinicopathological correlation and functional validation of specific long non-coding RNAs for hepatocellular carcinoma. Mol Cancer 16: 164, 2017.

29. Ma J, Zhang L, Yang P, Zeng ZC and Xiang ZL: Integrated analysis of long noncoding RNA expression profiles in lymph node metastasis of hepatocellular carcinoma. Gene 676: 47-55, 2018.

30. Zhang F, Wan M, Xu Y, Li Z, Kang P, Jiang X, Wang Y, Wang Z, Zhong $\mathrm{X}, \mathrm{Li} \mathrm{C}$, et al: Transcriptome analysis reveals dysregulated long non-coding RNAs and mRNAs associated with extrahepatic cholangiocarcinoma progression. Oncol Lett 14: 6079-6084, 2017.

31. Zhang W, Zhai Y, Wang W, Cao M and Ma C: Enhanced expression of lncRNA TP73-AS1 predicts unfavorable prognosis for gastric cancer and promotes cell migration and invasion by induction of EMT. Gene 678: 377-383, 2018.

32. Li C, Li W, Zhang Y, Zhang X, Liu T, Zhang Y, Yang Y, Wang L, Pan H, Ji J, et al: Increased expression of antisense lncRNA SPINT1-AS1 predicts a poor prognosis in colorectal cancer and is negatively correlated with its sense transcript. OncoTargets Ther 11: 3969-3978, 2018.

33. Jin L, He Y, Tang S and Huang S: LncRNA GHET1 predicts poor prognosis in hepatocellular carcinoma and promotes cell proliferation by silencing KLF2. J Cell Physiol 233: 4726-4734, 2018.

34. Carnero A, Garcia-Mayea Y, Mir C, Lorente J, Rubio IT and LLeonart ME: The cancer stem-cell signaling network and resistance to therapy. Cancer Treat Rev 49: 25-36, 2016.

35. Ravasio R, Ceccacci E and Minucci S: Self-renewal of tumor cells: Epigenetic determinants of the cancer stem cell phenotype. Curr Opin Genet Dev 36: 92-99, 2016.

36. Cardinale V, Renzi A, Carpino G, Torrice A, Bragazzi MC, Giuliante F, DeRose AM, Fraveto A, Onori P, Napoletano C, et al: Profiles of cancer stem cell subpopulations in cholangiocarcinomas. Am J Pathol 185: 1724-1739, 2015.

37. Kemmerling R, Alinger B, Dietze O, Bösmüller HC, Ocker M, Wolkersdörfer GW, Berr F, Neureiter D and Kiesslich T: Association of stem cell marker expression pattern and survival in human biliary tract cancer. Int J Oncol 41: 511-522, 2012.

38. Yoshikawa S, Zen Y, Fujii T, Sato Y, Ohta T, Aoyagi Y and Nakanuma Y: Characterization of $\mathrm{CD}_{133^{+}}$parenchymal cells in the liver: Histology and culture. World J Gastroenterol 15: 4896-4906, 2009.

This work is licensed under a Creative Commons Attribution-NonCommercial-NoDerivatives 4.0 International (CC BY-NC-ND 4.0) License. 